\title{
Existence and Uniqueness of Solution for Cahn-Hilliard Hyperbolic Phase-Field System with Dirichlet Boundary Condition and Regular Potentials
}

\author{
Jean De Dieu Mangoubi, Daniel Moukoko, Fidele Moukamba, Franck Davhys Reval Langa
}

G.R.A.F.E.D.P, Faculté des Sciences et Techniques, Université Marien NGOUABI, Brazzaville, Congo

Email: mangoubijean@gmail.com,moukokodani@yahoo.fr

How to cite this paper: De Dieu Mangoubi, J., Moukoko, D., Moukamba, F. and Langa, F.D.R. (2016) Existence and Uniqueness of Solution for Cahn-Hilliard Hyperbolic Phase-Field System with Dirichlet Boundary Condition and Regular Potentials. Applied Mathematics, 7, 1919-1926.

http://dx.doi.org/10.4236/am.2016.716157

Received: August 20, 2016

Accepted: October 11, 2016

Published: October 14, 2016

Copyright (c) 2016 by authors and Scientific Research Publishing Inc. This work is licensed under the Creative Commons Attribution International License (CC BY 4.0).

http://creativecommons.org/licenses/by/4.0/ (c) (i) Open Access

\begin{abstract}
Our aim in this paper is to study the existence and the uniqueness of the solutions for hyperbolic Cahn-Hilliard phase-field system, with initial conditions, Dirichlet boundary condition and regular potentials.
\end{abstract}

\section{Keywords}

Cahn-Hilliard Hyperbolic Phase-Field System, Regular Potential, Dirichlet Boundary Conditions

\section{Introduction}

G. Caginalp introduced in [1] the following phase-field system

$$
\begin{aligned}
& \frac{\partial u}{\partial t}-\Delta^{2} u-\Delta f(u)=-\Delta \theta \\
& \frac{\partial \theta}{\partial t}-\Delta \theta=-\frac{\partial u}{\partial t}
\end{aligned}
$$

where $u$ is the order parameter and $\theta$ is the (relative) temperature. These equations model phase transition processes such as melting-solidification processes and have been studied, see [2]-[6], for a similar phase-field model with a nonlinear term.

These Cahn-Hilliard phase-fiel system are known as the conserved phase-field system (see [7]-[9]) based on type III heat conduction and with two temperatures (see [10]). The authors have proved the existence and the uniqueness of the solutions, the existence of global attractor and of exponential attractors with singularly or regular 
potentials.

In [11], Ntsokongo and Batangouna have studied the following Cahn-Hilliard phasefield system

$$
\begin{aligned}
& \frac{\partial u}{\partial t}+\Delta^{2} u-\Delta f(u)=-\Delta\left(\frac{\partial \alpha}{\partial t}-\beta \Delta \frac{\partial \alpha}{\partial t}\right) \\
& \frac{\partial^{2} \alpha}{\partial t^{2}}-\Delta \frac{\partial^{2} \alpha}{\partial t^{2}}-\Delta \frac{\partial \alpha}{\partial t}-\Delta \alpha=-\frac{\partial u}{\partial t}
\end{aligned}
$$

where $\beta=1, u$ is the order parameter and $\alpha$ is the (relative) temperature, they have proved the existence and the uniqueness solution with Dirichlet boundary condition and regular potentials.

In this paper, we consider the following Cahn-Hilliard hyperbolic phase-fiel system

$$
\begin{aligned}
& \epsilon(-\Delta) \frac{\partial^{2} u}{\partial t^{2}}+\frac{\partial u}{\partial t}+\Delta^{2} u-\Delta f(u)=-\Delta \frac{\partial \alpha}{\partial t}, \\
& \frac{\partial^{2} \alpha}{\partial t^{2}}-\Delta \frac{\partial^{2} \alpha}{\partial t^{2}}-\Delta \frac{\partial \alpha}{\partial t}-\Delta \alpha=-\frac{\partial u}{\partial t}, \\
& \left.u\right|_{\partial \Omega}=\left.\alpha\right|_{\partial \Omega}=\left.\Delta u\right|_{\partial \Omega}=0, \\
& \left.u\right|_{t=0}=u_{0},\left.\frac{\partial u}{\partial t}\right|_{t=0}=u_{1},\left.\alpha\right|_{t=0}=\alpha_{0},\left.\frac{\partial \alpha}{\partial t}\right|_{t=0}=\alpha_{1},
\end{aligned}
$$

which is the perturbed phase-field system of Cahn-Hilliard phase-field system (3)-(4) with $\beta=0$. In the above hyperbolic system $\Omega$ is a bounded and regular domain of $\mathbb{R}^{n}$ with $n=2$ or 3 and $f$ is the nonlinear regular potentials.

The hyperbolic system has been extensively studied for Dirichlet boundary conditions and regular or singular potentials (see [12]-[14]). Whose certain have to end at existence of global attractor or at the existence of exponential attractors (see [15]).

In this paper we prove the existence and the uniqueness of solutions of (5)-(8). We consider the regular potential $f(s)=s^{3}-s$ which satisfies the following properties:

$$
\begin{aligned}
& f \text { is of class } C^{2} ; f(0)=0, \\
& -c_{0} \leq f^{\prime}(s), \quad c_{0} \geq 0, \quad \forall s \in \mathbb{R}, \\
& -c_{1} \leq F(s) \leq f(s) s+c_{2}, \quad c_{1}, c_{2} \geq 0, \quad \forall s \in \mathbb{R} \quad \text { where } F(s)=\int_{0}^{s} f(\tau) \mathrm{d} \tau .
\end{aligned}
$$

\section{Notations}

We denote by $\|$.$\| the usual L^{2}$-norm (with associated product scalar (...)) and set $\|\cdot\|_{-1}=\left\|(-\Delta)^{\frac{-1}{2}} \cdot\right\|$, where $-\Delta$ denotes the minus Laplace operator with Dirichlet boundary conditions. More generally, $\|\cdot\|_{X}$ denote the norm of Banach space $X$.

Throughout this paper, the same letters $c_{1}, c_{2}$ and $c_{3}$ denote (generally positive) constants which may change from line to line, or even a same line. 


\section{A Priori Estimates}

We multiply (5) by $(-\Delta)^{-1} \frac{\partial u}{\partial t}$ and (6) by $\frac{\partial \alpha}{\partial t}$, integrate over $\Omega$ and add the two resulting differential equalities. We find

$$
\frac{\mathrm{d} E_{1}}{\mathrm{~d} t}+2\left\|\frac{\partial u}{\partial t}\right\|_{-1}^{2}+2\left\|\nabla \frac{\partial \alpha}{\partial t}\right\|^{2}=0
$$

where

$$
E_{1}=\epsilon\left\|\frac{\partial u}{\partial t}\right\|^{2}+\|\nabla u\|^{2}+2 \int_{\Omega} F(u) \mathrm{d} x+\left\|\frac{\partial \alpha}{\partial t}\right\|^{2}+\left\|\nabla \frac{\partial \alpha}{\partial t}\right\|^{2}+\|\nabla \alpha\|^{2}
$$

satisfies

$$
E_{1} \geq C\left(\epsilon\left\|\frac{\partial u}{\partial t}\right\|^{2}+\|u\|_{H^{1}}^{2}+\left\|\frac{\partial \alpha}{\partial t}\right\|^{2}+\left\|\frac{\partial \alpha}{\partial t}\right\|_{H^{1}}^{2}+\|\alpha\|_{H^{1}}^{2}\right)+C^{\prime}, \quad C>0 .
$$

Finaly, we conclude that $u, \alpha \in L^{\infty}\left(\mathbf{R}^{+}, H_{0}^{1}(\Omega)\right)$,

$$
\frac{\partial u}{\partial t} \in L^{\infty}\left(\mathbf{R}^{+}, L^{2}(\Omega)\right) \cap L^{2}\left(0, T ; H^{-1}(\Omega)\right)
$$

and

$$
\frac{\partial \alpha}{\partial t} \in L^{\infty}\left(\mathbf{R}^{+}, H_{0}^{1}(\Omega)\right) \cap L^{2}\left(0, T ; H_{0}^{1}(\Omega)\right)
$$

for all $T>0$.

Multiply (6) by $\frac{\partial^{2} \alpha}{\partial t^{2}}$ and integrate over $\Omega$. We get.

$$
\begin{aligned}
& 2\left\|\frac{\partial^{2} \alpha}{\partial t^{2}}\right\|^{2}+2\left\|\nabla \frac{\partial^{2} \alpha}{\partial t^{2}}\right\|^{2}+\frac{\mathrm{d}}{\mathrm{d} t}\left\|\nabla \frac{\partial \alpha}{\partial t}\right\|^{2}=-2\left(\frac{\partial u}{\partial t}, \frac{\partial^{2} \alpha}{\partial t^{2}}\right)-2\left(\nabla \alpha, \nabla \frac{\partial \alpha}{\partial t}\right) \\
& \leq 2\left\|\frac{\partial^{2} \alpha}{\partial t^{2}}\right\|\left\|\frac{\partial u}{\partial t}\right\|+2\|\nabla \alpha\|\left\|\nabla \frac{\partial^{2} \alpha}{\partial t^{2}}\right\| \\
&\left\|\frac{\partial^{2} \alpha}{\partial t^{2}}\right\|^{2}+\left\|\nabla \frac{\partial^{2} \alpha}{\partial t^{2}}\right\|^{2}+\frac{\mathrm{d}}{\mathrm{d} t}\left\|\nabla \frac{\partial \alpha}{\partial t}\right\|^{2} \leq\left\|\frac{\partial u}{\partial t}\right\|^{2}+\|\nabla \alpha\|^{2} .
\end{aligned}
$$

Then $\frac{\partial^{2} \alpha}{\partial t^{2}} \in L^{2}\left(0, T ; H_{0}^{1}(\Omega)\right)$.

In this study, we have three main results; existence theorem, uniqueness theorem and existence theorem with more regularity.

\section{Existence and Uniqueness of Solutions}

Theorem 4.1. (Existence) We assume $\left(u_{0}, u_{1}, \alpha_{0}, \alpha_{1}\right) \in H_{0}^{1}(\Omega) \times L^{2}(\Omega) \times\left(H_{0}^{1}(\Omega)\right)^{2}$ then the system (5) - (8) possesses at least one solution $(u, \alpha)$ such that

$$
\begin{aligned}
& u, \alpha \in L^{\infty}\left(\mathbf{R}^{+}, H_{0}^{1}(\Omega)\right), \\
& \frac{\partial u}{\partial t} \in L^{\infty}\left(\mathbf{R}^{+}, L^{2}(\Omega)\right) \cap L^{2}\left(0, T ; H^{-1}(\Omega)\right),
\end{aligned}
$$




$$
\frac{\partial \alpha}{\partial t} \in L^{\infty}\left(\mathbf{R}^{+}, H_{0}^{1}(\Omega)\right) \cap L^{2}\left(0, T ; H_{0}^{1}(\Omega)\right)
$$

and $\frac{\partial^{2} \alpha}{\partial t^{2}} \in L^{2}\left(0, T ; H_{0}^{1}(\Omega)\right)$, for all $T>0$.

The proof is based on a priori estimates obtained in the previous section and on a standard Galerkin scheme.

Theorem 4.2. (Uniqueness) Let the assumpptions of Theorem 4.1 hold. Then, the system (5) - (8) possesses a unique solution $(u, \alpha)$ such that

$$
\begin{aligned}
& u, \alpha \in L^{\infty}\left(\mathbf{R}^{+}, H_{0}^{1}(\Omega)\right), \\
& \frac{\partial u}{\partial t} \in L^{\infty}\left(\mathbf{R}^{+}, L^{2}(\Omega)\right) \cap L^{2}\left(0, T ; H^{-1}(\Omega)\right), \\
& \frac{\partial \alpha}{\partial t} \in L^{\infty}\left(\mathbf{R}^{+} ; H_{0}^{1}(\Omega)\right) \cap L^{2}\left(0, T ; H_{0}^{1}(\Omega)\right)
\end{aligned}
$$

and $\frac{\partial^{2} \alpha}{\partial t^{2}} \in L^{2}\left(0, T ; H_{0}^{1}(\Omega)\right)$ for all $T>0$.

Proof. Let $\left(u^{(1)}, \alpha^{(1)}\right)$ and $\left(u^{(2)}, \alpha^{(2)}\right)$ be two solutions of the system (5)-(8) with initial data $\left(u_{0}^{(1)}, u_{1}^{(1)}, \alpha_{0}^{(1)}, \alpha_{1}^{(1)}\right)$ and $\left(u_{0}^{(2)}, u_{1}^{(2)}, \alpha_{0}^{(2)}, \alpha_{1}^{(2)}\right) \in H_{0}^{1}(\Omega) \times L^{2}(\Omega) \times\left(H_{0}^{1}(\Omega)\right)^{2}$, respectively. We set $u=u^{(1)}-u^{(2)}$ and $\alpha=\alpha^{(1)}-\alpha^{(2)}$, then $(u, \alpha)$ is solution of the following system

$$
\begin{aligned}
& \epsilon(-\Delta) \frac{\partial^{2} u}{\partial t^{2}}+\frac{\partial u}{\partial t}+\Delta^{2} u-\Delta\left(f\left(u^{(1)}\right)-f\left(u^{(2)}\right)\right)=-\Delta \frac{\partial \alpha}{\partial t}, \\
& \frac{\partial^{2} \alpha}{\partial t^{2}}-\Delta \frac{\partial^{2} \alpha}{\partial t^{2}}-\Delta \frac{\partial \alpha}{\partial t}-\Delta \alpha=-\frac{\partial u}{\partial t}, \\
& \left.u\right|_{\partial \Omega}=\left.\Delta u\right|_{\partial \Omega}=\left.\alpha\right|_{\partial \Omega}=0, \\
& \left.u\right|_{t=0}=u_{0}=u_{0}^{(1)}-u_{0}^{(2)},\left.\quad \frac{\partial u}{\partial t}\right|_{t=0}=u_{1}=u_{1}^{(1)}-u_{1}^{(2)} \\
& \left.\alpha\right|_{t=0}=\alpha_{0}=\alpha_{0}^{(1)}-\alpha_{0}^{(2)},\left.\quad \frac{\partial \alpha}{\partial t}\right|_{t=0}=\alpha_{1}=\alpha_{1}^{(1)}-\alpha_{1}^{(2)} .
\end{aligned}
$$

We multiply (12) by $(-\Delta)^{-1} \frac{\partial u}{\partial t}$ and integrate over $\Omega$. We find

$$
\frac{\mathrm{d}}{\mathrm{d} t}\left(\epsilon\left\|\frac{\partial u}{\partial t}\right\|^{2}+\|\nabla u\|^{2}\right)+2\left\|\frac{\partial u}{\partial t}\right\|_{-1}^{2}+2\left(f\left(u^{(1)}\right)-f\left(u^{(2)}\right), \frac{\partial u}{\partial t}\right)=2\left(\frac{\partial \alpha}{\partial t}, \frac{\partial u}{\partial t}\right) .
$$

Multiplying (13) by $\frac{\partial \alpha}{\partial t}$ and integrating over $\Omega$, we get

$$
\frac{\mathrm{d}}{\mathrm{d} t}\left(\left\|\frac{\partial \alpha}{\partial t}\right\|^{2}+\left\|\nabla \frac{\partial \alpha}{\partial t}\right\|^{2}+\|\nabla \alpha\|^{2}\right)+2\left\|\nabla \frac{\partial \alpha}{\partial t}\right\|^{2}=-2\left(\frac{\partial u}{\partial t}, \frac{\partial \alpha}{\partial t}\right) .
$$

Now summing (14) and (15) we obtain 


$$
\begin{aligned}
\frac{\mathrm{d} E_{2}}{\mathrm{~d} t}+2\left\|\frac{\partial u}{\partial t}\right\|_{-1}^{2}+2\left\|\nabla \frac{\partial \alpha}{\partial t}\right\|^{2} & =-2\left(f\left(u^{(1)}\right)-f\left(u^{(2)}\right), \frac{\partial u}{\partial t}\right) \\
& \leq\left\|f\left(u^{(1)}\right)-f\left(u^{(2)}\right)\right\|^{2}+\left\|\frac{\partial u}{\partial t}\right\|^{2},
\end{aligned}
$$

where

$$
E_{2}=\epsilon\left\|\frac{\partial u}{\partial t}\right\|^{2}+\|\nabla u\|^{2}+\left\|\frac{\partial \alpha}{\partial t}\right\|^{2}+\left\|\nabla \frac{\partial \alpha}{\partial t}\right\|^{2}+\|\nabla \alpha\|^{2} .
$$

Lagrange theorem gives a estimates

$$
\begin{aligned}
f\left(u^{(1)}\right)-f\left(u^{(2)}\right) & =\int_{0}^{1} f^{\prime}\left(u^{(2)}+s\left(u^{(1)}-u^{(2)}\right)\right) \mathrm{d} s u \\
& =\int_{0}^{1}\left(3\left(s u^{(1)}+(1-s) u^{(2)}\right)^{2}-1\right) \mathrm{d} s|u|,
\end{aligned}
$$

which implies

$$
\begin{aligned}
\left\|f\left(u^{(1)}\right)-f\left(u^{(2)}\right)\right\|^{2} & \leq 36 \int_{\Omega}\left(\left(u^{(2)}\right)^{2}+\left(u^{(1)}\right)^{2}+1\right)^{2}|u|^{2} \mathrm{~d} x \\
& \leq 36\left(\left\|u^{(2)}\right\|_{L^{6}}^{4}+\left\|u^{(1)}\right\|_{L^{6}}^{4}+1\right)\|u\|_{L^{6}}^{2} \\
& \leq C\left(\left\|\nabla u^{(2)}\right\|^{4}+\left\|\nabla u^{(1)}\right\|^{4}+1\right)\|\nabla u\|^{2} .
\end{aligned}
$$

Inserting the above estimate into (16), we have

$$
\frac{\mathrm{d} E_{2}}{\mathrm{~d} t}+2\left\|\frac{\partial u}{\partial t}\right\|_{-1}^{2}+2\left\|\nabla \frac{\partial \alpha}{\partial t}\right\|^{2} \leq K\left(\|\nabla u\|^{2}+\epsilon\left\|\frac{\partial u}{\partial t}\right\|^{2}\right), K>0 .
$$

Applying Gronwall's lemma, we obtain for all $t \in(0, T)$

$$
E_{2}(t)+2 \int_{0}^{t}\left(\left\|\frac{\partial u}{\partial t}(\tau)\right\|_{-1}^{2}+\left\|\nabla \frac{\partial \alpha}{\partial t}(\tau)\right\|^{2}\right) \mathrm{e}^{k(t-\tau)} \mathrm{d} \tau \leq E_{2}(0) \mathrm{e}^{k T} .
$$

We deduce the continuous dependence of the solution relative to the initial conditions, hence the uniqueness of the solution.

The existence and uniqueness of the solution of problem (5)-(8) being proven in a larger space, we will seek the solution with more regularity.

Theorem 4.3. Assume

$$
\left(u_{0}, u_{1}, \alpha_{0}, \alpha_{1}\right) \in\left(H^{2}(\Omega) \cap H_{0}^{1}(\Omega)\right) \times H_{0}^{1}(\Omega) \times\left(H^{2}(\Omega) \cap H_{0}^{1}(\Omega)\right)^{2},
$$

then the system (5)-(8) possesses a unique solution $(u, \alpha)$ such that

$$
\begin{aligned}
& u, \alpha \in L^{\infty}\left(0, T ; H^{2}(\Omega) \cap H_{0}^{1}(\Omega)\right), \\
& \frac{\partial u}{\partial t} \in L^{\infty}\left(0, T ; H_{0}^{1}(\Omega)\right) \cap L^{2}\left(0, T ; L^{2}(\Omega)\right), \\
& \frac{\partial \alpha}{\partial t} \in L^{\infty}\left(\mathbf{R}^{+}, H^{2}(\Omega) \cap H_{0}^{1}(\Omega)\right) \cap L^{2}\left(0, T ; H^{2}(\Omega) \cap H_{0}^{1}(\Omega)\right),
\end{aligned}
$$




$$
\frac{\partial^{2} \alpha}{\partial t^{2}} \in L^{2}\left(0, T ; H^{2}(\Omega) \cap H_{0}^{1}(\Omega)\right)
$$

and $\frac{\partial^{2} u}{\partial t^{2}} \in L^{2}\left(0, T ; L^{2}(\Omega)\right)$, for all $T>0$.

Proof. Following theorems 4.1 and 4.2, the system (5)-(8) possesses the unique solution $(u, \alpha)$ such that

$$
\begin{aligned}
& u, \alpha \in L^{\infty}\left(0, T ; H_{0}^{1}(\Omega)\right), \\
& \frac{\partial u}{\partial t} \in L^{\infty}\left(\mathbf{R}^{+}, L^{2}(\Omega)\right) \cap L^{2}\left(0, T ; H^{-1}(\Omega)\right), \\
& \frac{\partial \alpha}{\partial t} \in L^{\infty}\left(\mathbf{R}^{+}, H_{0}^{1}(\Omega)\right) \cap L^{2}\left(0, T ; H_{0}^{1}(\Omega)\right)
\end{aligned}
$$

and $\frac{\partial^{2} \alpha}{\partial t^{2}} \in L^{2}\left(0, T ; H_{0}^{1}(\Omega)\right)$, for all $T>0$.

Multiply (2.1) by $\frac{\partial u}{\partial t}$ and integrate over $\Omega$. We have

$$
\frac{\mathrm{d}}{\mathrm{d} t}\left(\epsilon\left\|\nabla \frac{\partial u}{\partial t}\right\|^{2}+\|\Delta u\|^{2}\right)+2\left\|\frac{\partial u}{\partial t}\right\|^{2}=2\left(\nabla \frac{\partial \alpha}{\partial t}, \nabla \frac{\partial u}{\partial t}\right)-2\left(\nabla f(u), \nabla \frac{\partial u}{\partial t}\right)
$$

we deduce the following inequality

$$
\frac{\mathrm{d}}{\mathrm{d} t}\left(\epsilon\left\|\nabla \frac{\partial u}{\partial t}\right\|^{2}+\|\Delta u\|^{2}\right)+2\left\|\frac{\partial u}{\partial t}\right\|^{2} \leq 2\left(\nabla \frac{\partial \alpha}{\partial t}, \nabla \frac{\partial u}{\partial t}\right)+2 \int_{\Omega}\left|f^{\prime}(u) \| \nabla u\right|\left|\nabla \frac{\partial u}{\partial t}\right| \mathrm{d} x .
$$

Thanks to use $f^{\prime}(s)$, we find the following estimate

$$
\begin{aligned}
2 \int_{\Omega}\left|f^{\prime}(u)\right||\nabla u|\left|\nabla \frac{\partial u}{\partial t}\right| \mathrm{d} x & \leq \int_{\Omega}\left|3 u^{2}-1\right||\nabla u|\left|\nabla \frac{\partial u}{\partial t}\right| \mathrm{d} x \\
& \leq \int_{\Omega}\left(3 u^{2}+1\right)|\nabla u|\left|\nabla \frac{\partial u}{\partial t}\right| \mathrm{d} x \\
& \leq C\left(\|u\|_{L^{6}}^{4}+1\right)\|\nabla u\|_{L^{6}}^{2}+\left\|\nabla \frac{\partial u}{\partial t}\right\|^{2} .
\end{aligned}
$$

Since $u \in L^{\infty}\left(0, T ; H_{0}^{1}(\Omega)\right)$, then the estimate (17) implies

$$
\frac{\mathrm{d}}{\mathrm{d} t}\left(\epsilon\left\|\nabla \frac{\partial u}{\partial t}\right\|^{2}+\|\Delta u\|^{2}\right)+2\left\|\frac{\partial u}{\partial t}\right\|^{2} \leq 2\left(\nabla \frac{\partial \alpha}{\partial t}, \nabla \frac{\partial u}{\partial t}\right)+C\|\Delta u\|^{2}+\left\|\nabla \frac{\partial u}{\partial t}\right\|^{2} .
$$

Multiplying (6) by $-\Delta \frac{\partial \alpha}{\partial t}$ and integrating over $\Omega$, we get

$$
\frac{\mathrm{d}}{\mathrm{d} t}\left(\left\|\nabla \frac{\partial \alpha}{\partial t}\right\|^{2}+\left\|\Delta \frac{\partial \alpha}{\partial t}\right\|^{2}+\|\Delta \alpha\|^{2}\right)+2\left\|\Delta \frac{\partial \alpha}{\partial t}\right\|^{2}=-2\left(\nabla \frac{\partial \alpha}{\partial t}, \nabla \frac{\partial u}{\partial t}\right) .
$$

Now summing (18) and (19), we obtain

$$
\frac{\mathrm{d} E_{3}}{\mathrm{~d} t}+2\left\|\frac{\partial u}{\partial t}\right\|^{2}+2\left\|\Delta \frac{\partial \alpha}{\partial t}\right\|^{2} \leq C\|\Delta u\|^{2}+\left\|\nabla \frac{\partial u}{\partial t}\right\|^{2}
$$

where 


$$
E_{3}=\epsilon\left\|\nabla \frac{\partial u}{\partial t}\right\|^{2}+\|\Delta u\|^{2}+\left\|\nabla \frac{\partial \alpha}{\partial t}\right\|^{2}+\left\|\Delta \frac{\partial \alpha}{\partial t}\right\|^{2}+\|\Delta \alpha\|^{2} .
$$

Appling the Gronwall's lemma, we deduce that $u, \alpha \in L^{\infty}\left(0, T ; H^{2}(\Omega) \cap H_{0}^{1}(\Omega)\right)$,

$$
\frac{\partial u}{\partial t} \in L^{\infty}\left(0, T ; H_{0}^{1}(\Omega)\right) \cap L^{2}\left(0, T ; L^{2}(\Omega)\right)
$$

and

$$
\frac{\partial \alpha}{\partial t} \in L^{\infty}\left(0, T ; H^{2}(\Omega) \cap H_{0}^{1}(\Omega)\right) \cap L^{2}\left(0, T ; H^{2}(\Omega) \cap H_{0}^{1}(\Omega)\right) .
$$

Multiplying (5) by $(-\Delta)^{-1} \frac{\partial^{2} u}{\partial t^{2}}$ and integrating ovre $\Omega$, we obtain

$$
\begin{aligned}
2 \epsilon\left\|\frac{\partial^{2} u}{\partial t^{2}}\right\|^{2}+\frac{\mathrm{d}}{\mathrm{d} t}\left\|\frac{\partial u}{\partial t}\right\|_{-1}^{2} & =2\left(\frac{\partial \alpha}{\partial t}, \frac{\partial^{2} u}{\partial t^{2}}\right)+2\left(\Delta u, \frac{\partial^{2} u}{\partial t^{2}}\right)-2\left(f(u), \frac{\partial^{2} u}{\partial t^{2}}\right), \\
& \leq 2\left\|\frac{\partial \alpha}{\partial t}\right\|\left\|\frac{\partial^{2} u}{\partial t^{2}}\right\|+2\|\Delta u\|\left\|\frac{\partial^{2} u}{\partial t^{2}}\right\|+2 \int_{\Omega}|f(u)| \frac{\partial^{2} u}{\partial t^{2}} \mid \mathrm{d} x .
\end{aligned}
$$

Thanks to use $f(s)$ and the fact that $u \in L^{\infty}\left(0, T ; H^{2}(\Omega) \cap H_{0}^{1}(\Omega)\right)$, we get

$$
\begin{aligned}
\int_{\Omega}|f(u)|\left|\frac{\partial^{2} u}{\partial t^{2}}\right| \mathrm{d} x & \leq\left\|u^{2}\right\|_{L^{\infty}} \int_{\Omega}|u|\left|\frac{\partial^{2} u}{\partial t^{2}}\right| \mathrm{d} x+\int_{\Omega}|u|\left|\frac{\partial^{2} u}{\partial t^{2}}\right| \mathrm{d} x \\
& \leq C\|\nabla u\|^{2}+\frac{\epsilon}{3}\left\|\frac{\partial^{2} u}{\partial t^{2}}\right\|^{2} .
\end{aligned}
$$

Inserting the above estimate into (20), we obtain

$$
\epsilon\left\|\frac{\partial^{2} u}{\partial t^{2}}\right\|^{2}+\frac{\mathrm{d}}{\mathrm{d} t}\left\|\frac{\partial u}{\partial t}\right\|_{-1}^{2} \leq C_{1}\left\|\frac{\partial \alpha}{\partial t}\right\|^{2}+C_{2}\|\Delta u\|^{2}+C_{3}\|\nabla u\|^{2}, C_{1}, C_{2}, C_{3}>0
$$

which implies that $\frac{\partial^{2} u}{\partial t^{2}} \in L^{2}\left(0, T ; L^{2}(\Omega)\right)$.

Multiplying (6) by $-\Delta \frac{\partial^{2} \alpha}{\partial t^{2}}$ and integrating over $\Omega$, we find

$$
2\left\|\nabla \frac{\partial^{2} \alpha}{\partial t^{2}}\right\|^{2}+\left\|\Delta \frac{\partial^{2} \alpha}{\partial t^{2}}\right\|^{2}+\frac{\mathrm{d}}{\mathrm{d} t}\left\|\Delta \frac{\partial \alpha}{\partial t}\right\|^{2} \leq 2\left\|\frac{\partial u}{\partial t}\right\|^{2}+2\|\Delta \alpha\|^{2}
$$

that implies $\frac{\partial^{2} \alpha}{\partial t^{2}} \in L^{2}\left(0, T ; H^{2}(\Omega) \cap H_{0}^{1}(\Omega)\right)$.

\section{Conclusion}

We have just shown the theorems of existence and uniqueness of the solutions for perturbed Cahn-Hilliard hyperbolic phase-field system with regular potentials.

\section{References}

[1] Caginalp, G. (1988) Conserved-Phase Field System: Implications for Kinetic Undercooling. Physical Review B, 38, 789-791. http://dx.doi.org/10.1103/PhysRevB.38.789

[2] Brochet, D., Hilhorst, D. and Novick-Cohen, A. (1996) Maximal Attractor and Inertial Sets 
for a Conserved Phase-Field Model. Advances in Differential Equations, 1, 547-578.

[3] Brochet, D. (1993) Maximal Attractor and Inertial Sets for Some Second and Fourth Order Phase-Field Models. In: Pitman Res. Notes Math. Ser, Vol. 296, Longman Sci. Tech., Harlow, 77-85.

[4] Colli, P., Gilardi, G., Grasselli, M. and Schimperna, G. (2001) The Conserved Phase-Field System with Memory. Adv. Math. Sci Appl., 11, 265-291.

[5] Gatti, S. and Pata, V. (2004) Exponential Attractor for a Conserved Phase-Field System with Memory. Physica D: Nonlinear Phenomena, 189, 31-48. http://dx.doi.org/10.1016/j.physd.2003.10.005

[6] Gilardi, G. (2007) On a Conserved Phase-Field Model with Irregular Potentials and Dynamic Boundary Condition. Rend. Cl. Sci. Mat. Nat., 141, 129-161.

[7] Miranville, A. (2013) On the Conserved Phase-Field Model. Journal of Mathematical Analysis and Applications, 400, 143-152. http://dx.doi.org/10.1016/j.jmaa.2012.11.038

[8] Caginalp, G. (1990) The Dynamic of Conserved Phase Field System: Stefan-Like, HeleShaw and Cahn-Hilliard Models as Asymptotic Limits. IMA Journal of Applied Mathematics, 44, 77-94.

[9] Colli, P., Gilardi, G., Laurenot, Ph. and Novick-Cohen, A. (1999) Uniqueness and LongTime Behavior for the Conserved Phase-Field System Memory. Discrete and Continuous Dynamical Systems—Series A, 5, 375-390. http://dx.doi.org/10.3934/dcds.1999.5.375

[10] Miranville, A. and Quintanilla, R. (2011) A Type III Phase-Field System with a Logarithmic Potential. Applied Mathematics Letters, 24, 1003-1008. http://dx.doi.org/10.1016/j.aml.2011.01.016

[11] Ntsokongo, A.J. and Batangouna, N. (2016) Existence and Uniqueness of Solutions for a Conserved Phase-Field Type Model. AIMS Mathematics, 1, 144-155.

http://dx.doi.org/10.3934/Math.2016.2.144

[12] Goyaud, M.E.I., Moukamba, F., Moukoko, D. and Langa, F.D.R. (2015) Existence and Uniqueness of Solution for Caginalp Hyperbolic Phase Field System with Polynomial Growth Potential. International Mathematical Forum, 10, 477-486.

[13] Moukoko, D. (2014) Well-Posedness and Longtime Behaviors of a Hyprebolic Caginalp System. Journal of Applied Analysis and Computation, 4, 151-196.

[14] Moukoko, D. (2015) Etude de Modeles Hyperboliques de champ de phase de Caginalp, These unique, Falculté des Sciences et Techniques, Université Marien NGOUABI.

[15] Moukoko, D., Moukamba, F. and Reval, L.F.D. (2015) Global Attractor for Caginalp Hyperbolics Field-Phase System with Singular Potential. Journal of Mathematics Research, 7, 165-177. 
Submit or recommend next manuscript to SCIRP and we will provide best service for you:

Accepting pre-submission inquiries through Email, Facebook, LinkedIn, Twitter, etc. A wide selection of journals (inclusive of 9 subjects, more than 200 journals)

Providing 24-hour high-quality service

User-friendly online submission system

Fair and swift peer-review system

Efficient typesetting and proofreading procedure

Display of the result of downloads and visits, as well as the number of cited articles

Maximum dissemination of your research work

Submit your manuscript at: http://papersubmission.scirp.org/

Or contact am@scirp.org 